\title{
Epidemiological characteristics of pertussis in Estonia, Lithuania, Romania, the Czech Republic, Poland and Turkey-1945 to 2005
}

\author{
Irja Lutsar • Ioana Anca • Mustafa Bakir • \\ Vytautas Usonis • Roman Prymula • Nuran Salman • \\ Pawel Grezesiowski • Michael Greenberg • \\ on behalf of the Central European Vaccination Advisory \\ Group (CEVAG)
}

Received: 24 January 2008 /Revised: 4 April 2008 / Accepted: 22 April 2008 / Published online: 5 July 2008

(C) Springer-Verlag 2008

\begin{abstract}
Pertussis epidemiology was examined in selected Central and Eastern European countries andTurkey (CEEs) from 1945 to 2005. Epidemiology and immunisation coverage data were collected fromNational Health Departments
\end{abstract}

Sources of support This review of data was produced with the financial support of GlaxoSmithKline Biologicals.

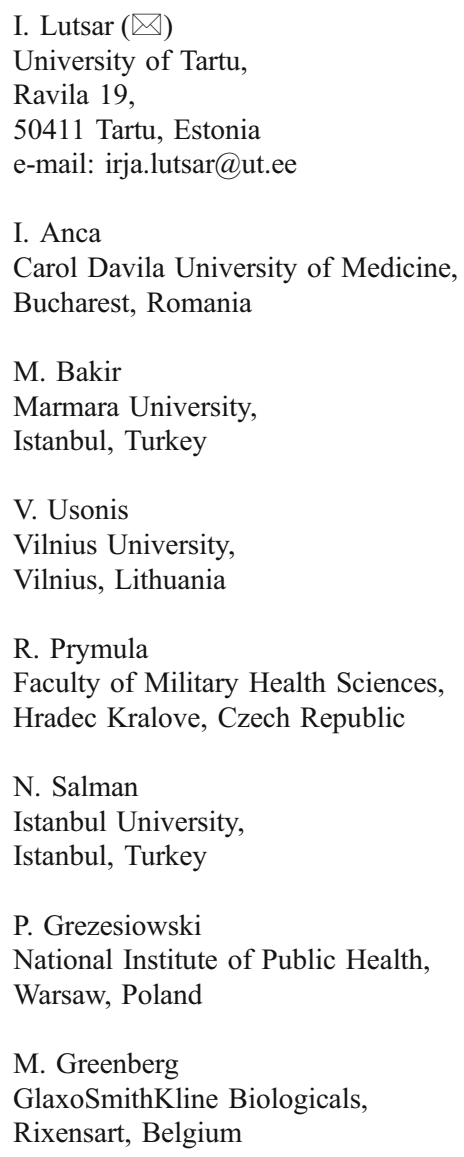

and Epidemiology Institutes. Pertussis diagnosis was made by the World Health Organization (WHO) clinical criteria, laboratory confirmation and/or epidemiological link, except for Romania (WHO clinical case definition used). In the pre-vaccine era, pertussis incidence (except Turkey) exceeded 200/100,000 (range180-651/100,000), with 60$70 \%$ of cases occurring in pre-school children. Until 2007, a second-year booster was givenin Estonia, Lithuania and Turkey, and an additional pre-school booster elsewhere. During 1995-2005, immunisation coverage by the age of 2 years exceeded $80 \%$ (range $80-98 \%$ ) and, excluding Estonia, pertussis incidence was $<3 / 100,000$. Age-specific incidence rates rose in 5-14 year olds in Poland, Estonia and the Czech Republic. Incidence rates in children $<1$ year of age remained unchanged. There were two age distribution patterns. In the Czech Republic and Estonia, 16\% of cases occurred in pre-school children and $17 \%$ and $22 \%$ in children $>15$ years of age, respectively; in Romania, Turkey and Lithuania, $51 \%, 71 \%$ and $73 \%$, respectively, occurred in pre-school children and $<7 \%$ in children aged $>15$ years. Pertussis infection persists, despite high immunisation coverage. Compared with the pre-vaccine era, the age distribution changed differentially in CEEs, with an apparent shift towards older children.

Keywords Pertussis $\cdot$ Epidemiology $\cdot$ Europe $\cdot$ Vaccination
Abbreviations
DTP Diphtheria-tetanus-pertussis (acellular or whole- cell) vaccine
CEEs Central and Eastern European countries and Turkey
CEVAG Central European Vaccination Advisory Group 
PCR Polymerase chain reaction

PT Pertussis toxin

USA United States of America

\section{Introduction}

Approaches to pertussis diagnosis, surveillance and immunisation vary widely across Europe. However, most countries have reported high levels of vaccine coverage in infants and toddlers, and significant reductions in infant morbidity and mortality have been achieved [19]. Although the absolute incidence of pertussis has substantially decreased due to the effective protection of infants and toddlers, the relative proportion of older age groups with pertussis, adolescents and adults in particular, has recently increased in several countries, including Europe, the United States (USA) and Canada [2, 5, 8, 12, 19]. A substantial disease burden also persists in these countries amongst infants too young to be fully vaccinated $[5,8]$. In Sweden, the overall disease rates in very young infants fell after the introduction of routine pertussis vaccination. However, since 2001, the disease rates in this group remain higher than in any other age group [6].

The epidemiology of pertussis in Western European countries was recently reviewed by Celentano et al. [1], who noted that pertussis is still an endemic disease in most countries and that, between 1998 and 2002, the reported incidence rates in the $>14$ years old age group increased by $115 \%$. A comparison of pertussis surveillance systems in European countries has recommended that the World Health Organization (WHO) case definition, the standardisation of laboratory diagnosis and the integration of information on deaths from alternative sources should be supported [16]. However, the epidemiology of pertussis in Eastern Europe has, thus far, been reported only on an individual country basis [7, 15].

This report is based on pertussis epidemiology and the data presented by countries present at a meeting of the Central European Vaccination Advisory Group (CEVAG) which took place in Bucharest, Romania, in March 2006. The CEVAG was established in 2005 to address issues relating to the impact, control and prevention of vaccinepreventable diseases in Central Europe [3]. Its members include vaccinologists, epidemiologists and paediatricians. We describe pertussis epidemiology and vaccination coverage rates in selected Central and Eastern European countries and Turkey (CEEs) from 1945 to 2005 obtained through a retrospective review of existing data sources in the National Health Departments and Epidemiology Institutes of selected CEE countries.

\section{Materials and methods}

Data sources

A structured request for data was sent to CEVAG members from each country to gather as close to a uniform set of data as possible from each country. These data were presented at the CEVAG meeting in March 2006. The countries included in this analysis were: Estonia, Lithuania, Poland, the Czech Republic, Romania and Turkey. The national surveillance systems in all of the participating countries collected casebased information on pertussis through statutory notifications by medical practitioners, and covered all ages.

In Estonia, the data were retrieved from the Health Protection Inspectorate; the reporting of suspected and/or confirmed pertussis cases has been mandatory since 1945 . In Lithuania, the data were retrieved from the Centre of the Communicable Diseases Prevention and Control and in Poland from the National Register of Infectious Disease maintained by Sanitary Inspection.

In the Czech Republic, the data were accessed from the National Institute of Public Health. In the Czech Republic, pertussis reporting was mandatory only from institutional care facilities. Surveillance tools have altered periodically since 1945 (the Information System on Communicable Diseases [ISPO] between 1982 and 1992, and EPIDAT from 1993 to the present). However, in all systems, local reports of pertussis disease were provided to the district level, district level to regional level and from regional to national level.

The data in Romania were collected from the Romanian Ministry of Public Health. Surveillance is conducted by the National Surveillance Programme for Pertussis and the Cantacuzino Institute - National Reference Centre for Pertussis. Romanian health legislation requires mandatory hospital admission for all paediatric pertussis cases (suspected or confirmed).

In Turkey, the data were accessed from the Turkish Ministry of Health. Reports of pertussis disease were required to be reported to the Department of Health office in every district by a phone service available 24 hours a day. A case investigation form was to be completed in every case and clinical samples were sent to the reference microbiology laboratory for culture.

The following data are presented from 1945 through 2005: total yearly incidence rates, age-specific incidence rates, mortality rates, age distribution and the percentage of children immunised with three doses of diphtheria-tetanus-pertussis (whole-cell or acellular) (DTP) vaccine by the age of two years.

Case definition

The WHO clinical case definition for pertussis was used in all countries, defined as a case diagnosed as pertussis by a 
Table 1 Immunisation schedule by country in 2005

\begin{tabular}{|c|c|c|c|c|}
\hline & $\begin{array}{l}\text { Year when immunisation } \\
\text { was initiated }\end{array}$ & Primary immunisation & First booster & $* *$ Second booster \\
\hline Estonia & 1957 & $3 \mathrm{~m}, 4.5 \mathrm{~m}, 6 \mathrm{~m}$ & $24 \mathrm{~m}$ & - \\
\hline Poland & 1960 & $6 \mathrm{w}, 12 \mathrm{w}, 18 \mathrm{w}$ & $18 \mathrm{~m}$ & *6y \\
\hline Lithuania & 1956 & $2 \mathrm{~m}, 4 \mathrm{~m}, 6 \mathrm{~m}$ & $18 \mathrm{~m}$ & - \\
\hline Romania & 1961 & $2 \mathrm{~m}, 4 \mathrm{~m}, 6 \mathrm{~m}$ & $12 \mathrm{~m}$ & $30-35 \mathrm{~m}$ \\
\hline Czech Republic & 1958 & $3 \mathrm{~m}, 4 \mathrm{~m}, 5 \mathrm{~m}$ & $18-20 \mathrm{~m}$ & $* 5 y$ \\
\hline Turkey & 1968 & $2 \mathrm{~m}, 3 \mathrm{~m}, 4 \mathrm{~m}$ & $16-24 \mathrm{~m}$ & - \\
\hline
\end{tabular}

*Whole-cell vaccines were used in most countries except at the age points marked with asterisks, where acellular vaccines were used

**A second booster was given in Poland from 2004, in Romania from 1961 and in the Czech Republic at the age of 6 years between 1958 and 1994 and at the age of 5 years from 1995

physician or a person with a cough lasting at least two weeks with at least one of the following symptoms: paroxysms of coughing, inspiratory whooping or post-tussive vomiting without other apparent cause [20]. In addition, in all countries except Romania, laboratory confirmation and/ or epidemiological link with a confirmed case was also required. The available laboratory tests included culture, serology (except Turkey) and, more recently, polymerase chain reaction (PCR) (Estonia, Czech Republic, Turkey). Details of the serology tests used in each laboratory over time were not available in full. In Estonia and Lithuania, the serological confirmation of pertussis disease is defined as pertussis toxin (PT) IgA or IgM levels $>12$ EU (enzymelinked immunosorbent assay [ELISA] units) or a four-fold increase in paired sera collected 10-14 days apart. In the Czech Republic, pertussis disease cases were confirmed by positive IgG PT in paired samples. In Poland, cases were confirmed by elevated PT IgA. In Romania, tests for specific antibodies against pertussis used agglutination and complement fixing reactions.

\section{Data analysis}

The median incidence and mortality rates per 100,000 population were calculated for the following periods: preor early vaccine era (1945-1960) and then 1961-1975 (era characterised by widespread vaccination in most countries),
1976-1990 (era when vaccination in the former Soviet Union became unpopular and the rates dropped dramatically) and 1991-2005 (post-Soviet Union era). The following age categories were considered for the analysis: $<1$ years, $1-4$ years, $5-9$ years, $10-14$ years and $\geq 15$ years.

Pertussis vaccines and vaccination schedules

The immunisation schedules and type of vaccines used (whole-cell versus acellular) were obtained from National Public Health Departments, Epidemiology Institutes and/or from vaccine wholesalers in the participating countries.

\section{Results}

Recent vaccination schedules for the countries studied are presented in Table 1. In all countries, acellular vaccines were available on the private market. Data obtained from each country on the primary vaccination coverage rates by the age of two years available from 1991 onwards are presented in Table 2. These data are consistent with DTP3 coverage reported by the WHO [21]. The overall incidence rates for pertussis infections in the countries studied from 1945 to 2005 are shown in Fig. 1.

With the exception of Turkey, almost half of all pertussis cases occurred in school-aged children in all countries,

Table 2 Percentage of children immunised (IR) at the age of two and the incidence (Inc) of pertussis per 100,000 population in the period 1991-2005

\begin{tabular}{|c|c|c|c|c|c|c|c|c|c|c|c|c|}
\hline & \multicolumn{2}{|l|}{ Estonia } & \multicolumn{2}{|l|}{ Poland } & \multicolumn{2}{|c|}{ Lithuania } & \multicolumn{2}{|c|}{ Romania } & \multicolumn{2}{|c|}{ Czech Republic } & \multicolumn{2}{|l|}{ Turkey } \\
\hline & IR $(\%)$ & Inc & IR $(\%)$ & Inc & IR $(\%)$ & Inc & IR $(\%)$ & Inc & IR (\%) & Inc & IR $(\%)$ & Inc \\
\hline 1991-1995 & 77 & 12.0 & 94 & 1.4 & 83 & 6.8 & 98 & 4 & 99 & 0.3 & 78 & 0.7 \\
\hline 1996-2000 & 88 & 23.0 & 97 & 5.9 & 93 & 2.5 & 98 & 1 & 98 & 1.1 & 80 & 0.8 \\
\hline 2001-2005 & 94 & 23.3 & 98 & 5.3 & 94 & 1.4 & 97 & 0.7 & 98 & 3.4 & 83 & 0.3 \\
\hline Median & 88 & 16.2 & 98 & 5.1 & 93 & 2.5 & 98 & 1.5 & 98 & 1.1 & 80 & 0.6 \\
\hline
\end{tabular}


Fig. 1 Incidence of pertussis in various countries from 1945 to 2005

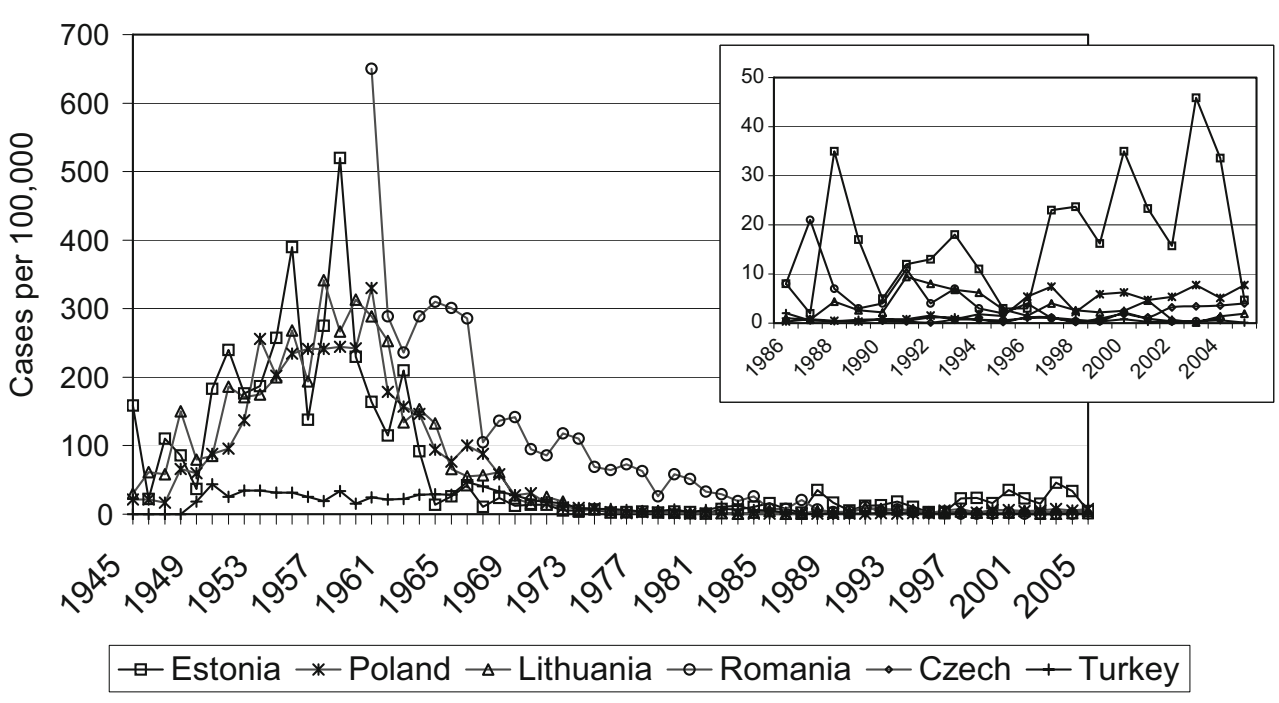

irrespective of whether a second booster was used or not. No correlation was noted between the age distribution of pertussis infection and the use of a school entry pertussis booster vaccination.

\section{Czech Republic}

Vaccination against pertussis began in the Czech Republic in 1958. TetrACT-HIB ${ }^{\text {TM }}$ (Sanofi Pasteur, Lyon, France) is used for primary vaccination and the first booster dose, whereas the second booster dose is given using acellular vaccines. Consistently high levels of vaccine coverage have been achieved (Table 2). Incidence rates (data available from 1990) were low $(<1 / 100,000$, Fig. 1) until 1994, when an increase was observed affecting the 5 to 14 year old age group (Fig. 2). The incidence rates remained unchanged in infants aged $<1$ year. Between 1991 and 2005, the highest proportion of cases was observed in individuals $>15$ years of age (Fig. 3).

\section{Estonia}

Vaccination against pertussis began in Estonia in 1957, with increasing vaccine coverage over the last 15 years (Table 2). The whole-cell vaccines produced in the Research Institutes of Moscow were used from 1957 to 1965, an adsorbed DTPw vaccine (produced in the Soviet Union) from 1966 to 1991 and TetrACT-HIB ${ }^{\text {TM }}$ has been in use since 1992. Over the years, the immunisation schedule has been similar to that presented in Table 1, except for the second booster that was given at school entry from 1964 to 1980 . An increase in the total pertussis incidence was observed during this period in Estonia following a steady decrease in pertussis observed prior to 1991 (Fig. 1). Between 1991 and 2005, the overall incidence rate of pertussis was between 12.0 and 23.3 per 100,000 population, which is considerably higher than the rates observed in other countries (Table 2 ). The mortality rate in the pre-vaccine era was 0.5 per 100,000 population. No deaths were reported between 1991 and 2005. Age-specific pertussis disease incidence rates for Estonia over the last 15 years showed a consistent increase in incidence rates for the between 5 and 14 years old age group, while the incidence rates in $<1$ year olds remained unchanged (Fig. 2). A clear shift was seen over time towards a higher ratio of cases in older age groups (Fig. 4) [11], with nearly $20 \%$ of cases occurring in those $>15$ years of age (Fig. 3).

\section{Lithuania}

Vaccination against pertussis began in Lithuania in 1956. Whole-cell vaccine (TetrACT-HIB ${ }^{\mathrm{TM}}$ ) is used for primary and booster doses. Over the last 15 years, the vaccination rates have increased in Lithuania (Table 2), while pertussis disease incidence decreased progressively between 1945 and 2005 (Fig. 1). The age distribution data for pertussis infection available from 1991 to 2005 showed that, in Lithuania, the majority of pertussis cases occurred in infants $<1$ year of age, with minimal pertussis reported in subjects $>15$ years of age (Fig. 3).

\section{Poland}

Vaccination against pertussis began in Poland in 1960. Primary vaccination doses and the first booster dose are provided using adsorbed DTP vaccine, IBSS Biomed (Krakow, Poland) (Table 1). The second booster is provided using acellular vaccines. In the last 15 years, pertussis incidence increased (Fig. 1), mainly in the 5 to 14 year old age group (Fig. 2). The incidence among infants remained unchanged. However, the highest number of cases continued to occur at age $<1$ year (Fig. 3). The mortality rate in the pre-vaccine era was 2.3 per 100,000 in Poland. During the last 15 years, no deaths were reported. 
Fig. 2 Age-dependent

incidence of pertussis infection in the Czech Republic during the period 1993-2005 (A),

Estonia 1991-2005 (B) and

Poland 1990-2003 (C)

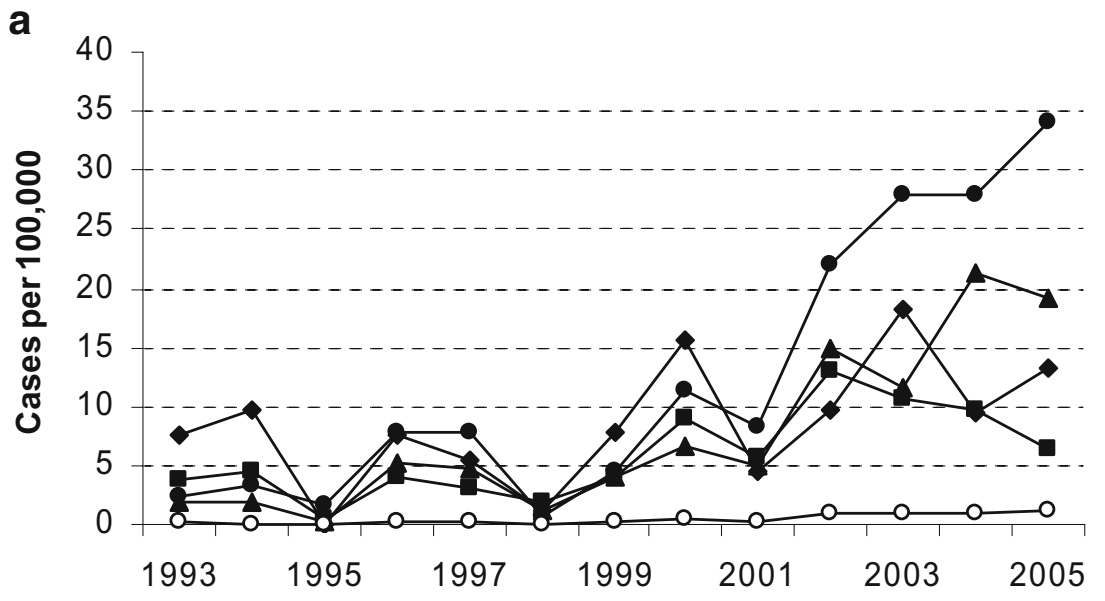

$\multimap<1$ year $\rightarrow-1-4$ years $\multimap 5-9$ years $\multimap-10-14$ years $\multimap>15$ years

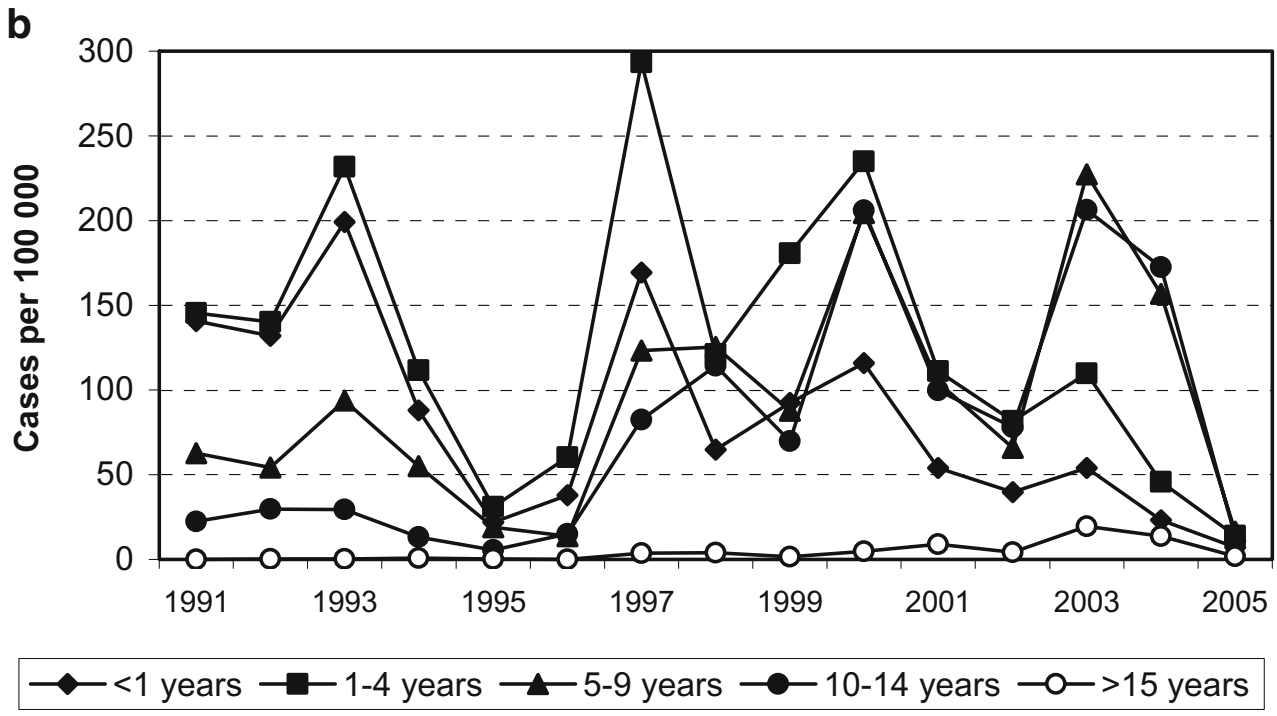

C

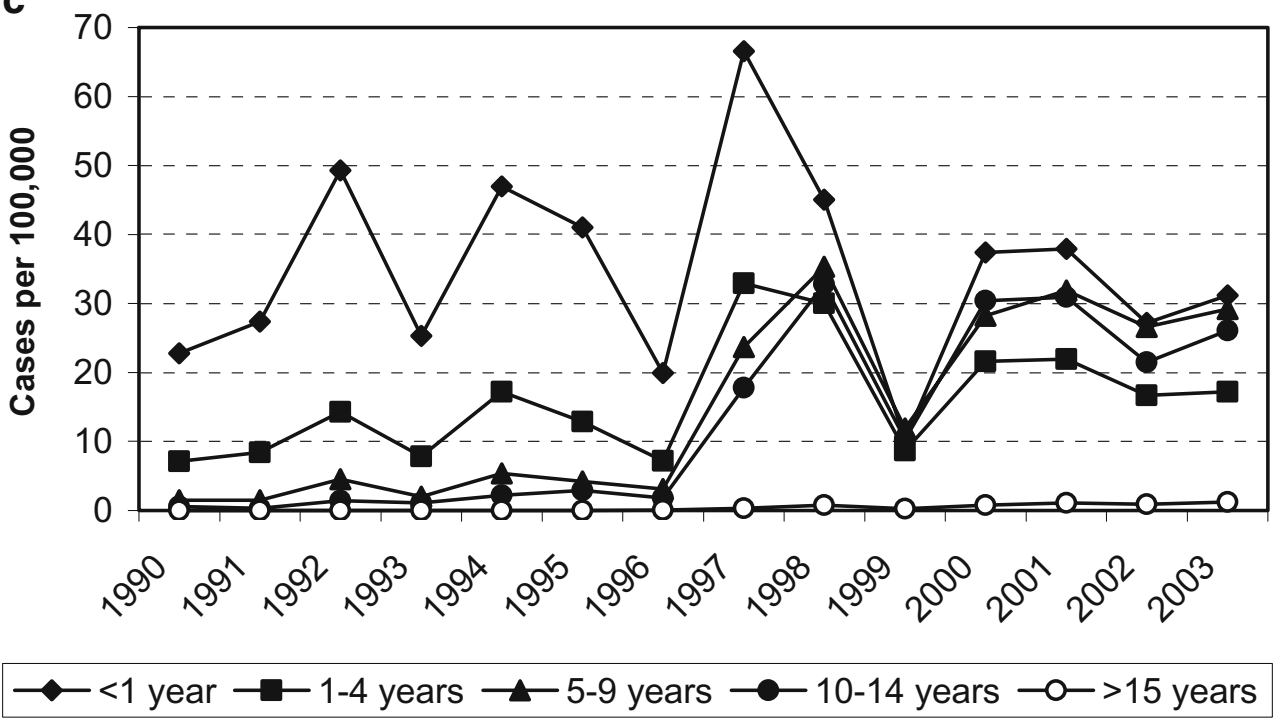


Fig. 3 Proportion (\%) of various age groups among pertussis patients in different countries in 1991-2005. ${ }^{\mathrm{a}}$ For Lithuania, the percentages are given for the 3-6 years old $(24.9 \%)$ and $7-14$ years old $(27.6 \%)$ age groups. ${ }^{\mathrm{b}}$ Represents age $<2$ years. The other age groups were not done

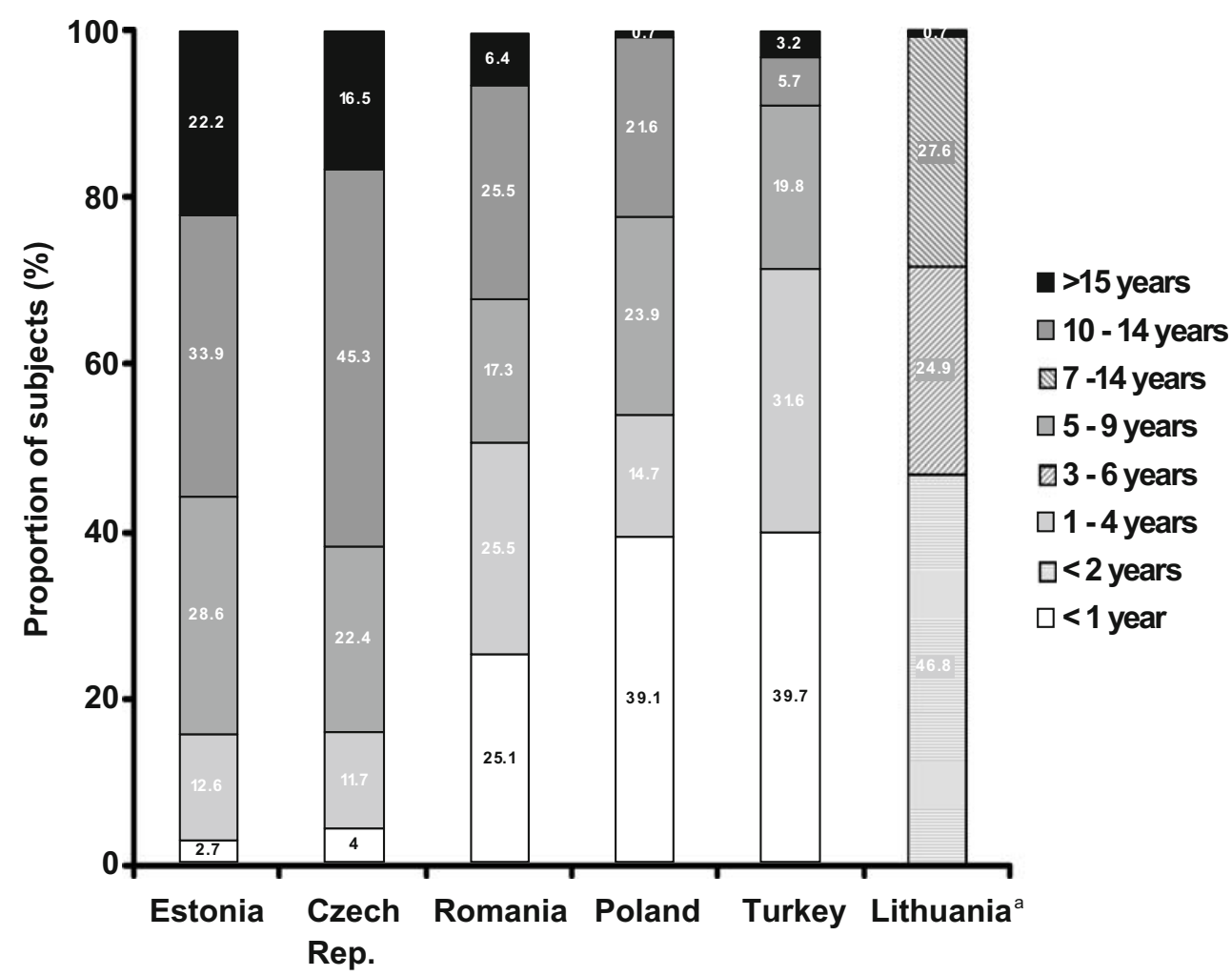

\section{Romania}

Vaccination against pertussis began in Romania in 1961. DT COQ ${ }^{\mathrm{TM}}$ (Sanofi Pasteur, France) and Tritanrix ${ }^{\mathrm{TM}} \mathrm{HepB}$ (GlaxoSmithKline Biologicals, Rixensart, Belgium) are used for the primary and booster doses in Romania. Vaccination coverage rates have remained largely unchanged over time (Table 2). Prior to the introduction of immunisation, the median pertussis incidence rate reached $650 / 100,000$ (Fig. 1), with a mortality of 4.85 per 100,000 population. A steady reduction in incidence rates was observed over the next four decades. Pertussis mortality in Romania between 1991 and 2005 did not exceed 0.01/ 100,000 . The age distribution of pertussis disease has remained constant. The highest number of cases occurred at age $<1$ year (Fig. 3).

\section{Turkey}

Vaccination against pertussis began in Turkey in 1968. DTP vaccine adsorbed (Serum Institute of India, Pune, India) is used for the primary and booster doses. Vaccination coverage remained constant ( $78 \%$ to $83 \%$ ) between 1991 and 2005. Pertussis incidence in the pre-vaccine era was lower in Turkey than the other countries described above (Fig. 1). However, a steady decline in the incidence rates after the introduction of vaccination was similarly observed. During the last 15 years, the median mortality rate in
Turkey was 0.002 per 100,000 . No change in the age distribution of cases has been observed in the last 15 years. The highest number of cases occurred in infants age $<1$ year (Fig. 3).

\section{Discussion}

To our knowledge, this is the first review on the epidemiology of pertussis in selected Central and Eastern European countries over the last 60 years, a time during which these countries underwent major economical and political changes. This review has highlighted that, with the introduction of immunisation in the late 1950s and early 1960s, the incidence of pertussis has dropped significantly and mortality has become almost non-existent in CEEs. However, in the last 15 years, in some countries (e.g. Estonia and Poland), pertussis incidence has increased in a similar fashion to the increases noted in the USA $[5,9]$, with the age distribution of pertussis infection apparently shifting towards older children. Although not observed in our study, a similar increase affecting adolescents has also been reported recently in Turkey [4].

Similarly to results from a review of pertussis epidemiology conducted in Western Europe, the incidence rates of pertussis observed in the countries presented in this report were variable: they were higher in most Northern countries, particularly in Estonia, while lower rates were observed in Southern countries, such as Romania and Turkey [1]. 
Fig. 4 Age distribution of pertussis patients from 1948 to 2004 in Estonia. A Age as a proportion of all pertussis cases. B Rate per 100,000 population

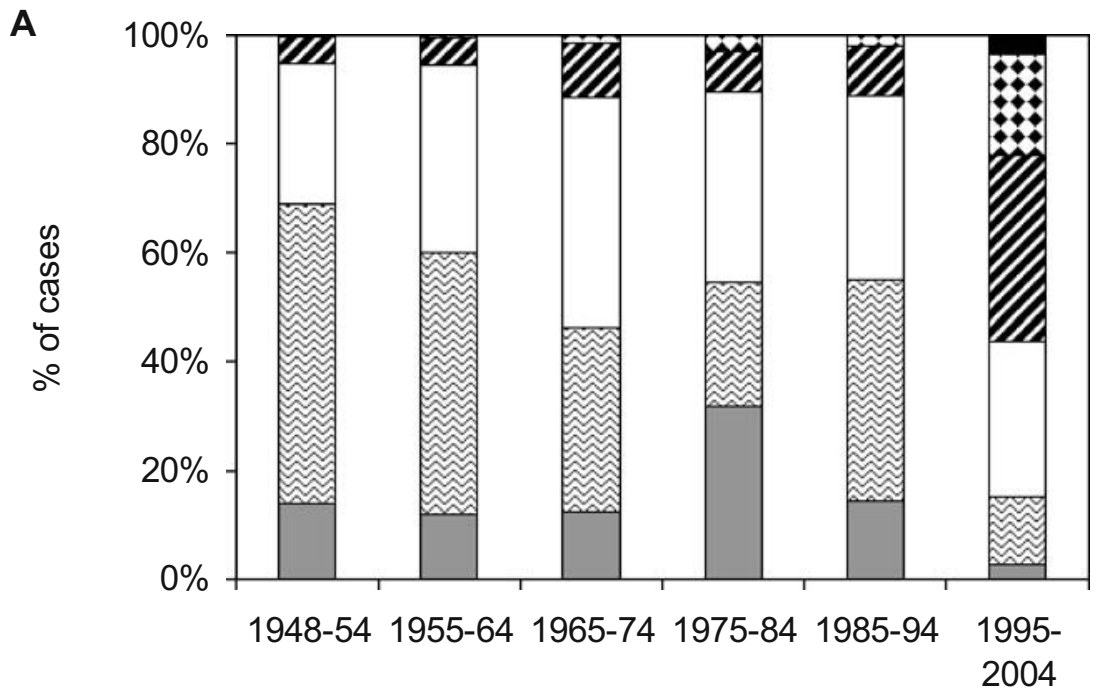

$\square<1$ year 图 1-4 years $\square$ 5-9 years $\square 10-14$ years $\mathbf{Z} 15-50$ years

$>50$ years

B

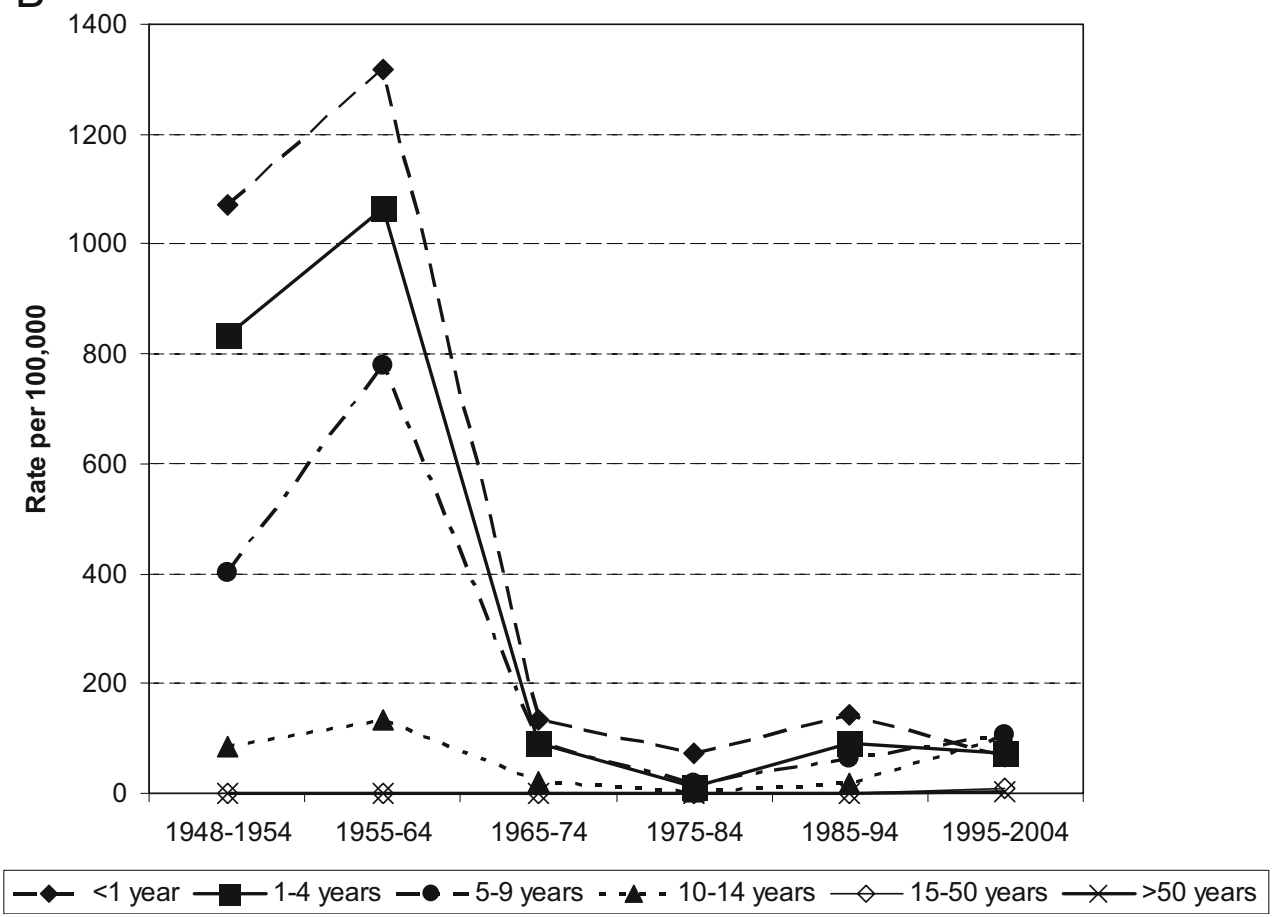

A significant limitation of this review is its retrospective nature. Data were collected from national registration systems into which primary care physicians reported pertussis cases, but the completeness of such data is not systematically checked. Furthermore, although pertussis cases with serological confirmation were included in the analysis for all countries except Turkey, the details of the serological tests and cut-offs used during the 60 years of the study were not available in full.
Another important limitation of the study is that of pertussis under diagnosis and under reporting. Under diagnosis and under reporting probably arise from both incomplete use of bacterial culture techniques and PCR by primary care physicians and, we expect, by a considerable degree of under reporting in the older age groups, in whom pertussis is less likely to be considered as a possible cause of cough illness.

This hypothesis is supported by evidence from Turkey and Lithuania. In a Turkish seroprevalence study, IgG 
antibodies against pertussis were present only in $1 / 3$ of subjects at the age of 0-73 months, while from 12 to 17 years of age, almost all subjects had anti-PT and anti-FHA antibodies, despite the fact that no second pertussis booster was given $[10,17]$. Such high seroprevalence rates among adolescents indicate widespread natural contacts with Bordetella pertussis and suggest that the incidence rates of pertussis in Turkey are much higher than that reported in the national surveillance system and, thus, those presented here. A study conducted in Lithuania also suggested that pertussis infection is more common in older age groups than has been officially reported [13]. The under reporting of pertussis among adolescents and adults in the USA was confirmed by another recent study reporting an incidence rate of approximately $1 \%$ in this population, which is much higher than that reported here [18].

National programmes for primary immunisation in all of the countries presented in this review provide whole-cell pertussis vaccines. However, over the last decade, the introduction of acellular vaccines on the private markets has occurred. The size of the latter is not fully characterised but, according to the wholesale figures of 2005 in Estonia and the Czech Republic, up to $1 / 4$ of infants have been immunised with acellular vaccines. In 2007, acellular vaccines have been introduced into the national programme in Lithuania and in the Czech Republic.

Although the introduction of immunisation programmes clearly resulted in a drop in morbidity rates in all countries (except Turkey, in which reporting problems may have predated vaccine introduction [personal communication from the Turkish Ministry of Health]), we were unable to demonstrate any correlation between the primary immunisation rates and the overall incidence of pertussis. There are several reasons for this. Firstly, the reported incidence rates are based on passive notification and, therefore, cannot be interpreted as an accurate reflection of the true incidence of infection or disease in these populations. Secondly, in the past 10 years, the immunisation rates in all countries except for Estonia and Turkey have been over $95 \%$ and, thus, minor improvements may have had barely any noticeable influence on the incidence of disease. Thirdly, a closer look at the Estonian data clearly demonstrates that, with the improved primary immunisation coverage, the incidence of pertussis in infancy has dropped. Finally, the primary immunisation rates are unlikely to affect disease rates in school-aged children or adults, since protection against pertussis disease is not life-long.

Despite the likely under reporting, an increased incidence of infant, adolescent and adult pertussis has been observed worldwide, in spite of the widespread introduction of vaccination programmes [14]. This is a cause of concern because adolescents and adults have been identified as a source of transmission of pertussis to very young infants who have not been immunised or who are only partially immunised, and, thus, more vulnerable to disease-related complications and mortality. A few countries, including the USA, Australia, Austria, Canada, France and Germany, have incorporated an adolescent booster dose into their immunisation schedules, in recognition of the changing age distribution of pertussis infection [14].

In conclusion, on the basis of the currently available epidemiologic data, it appears that there is a need for the improved recognition, diagnosis and reporting of pertussis in Central and Eastern European countries, in particular, among older children and in adults. In view of the shift in age-specific incidence rates towards older children and adolescents in some CEEs, consideration should be given to the incorporation of additional booster doses of pertussis vaccine into national vaccination schedules. Our review has provided useful insight about similarities between certain countries with similar vaccination programmes, enabling recommendations to be made on the basis of data generated in countries with similar epidemiologies.

Acknowledgements The authors wish to thank the staff of the national surveillance systems for providing the data. Mr. Simon Lancaster, Dr. Joanne Wolter and Dr. Véronique Delpire helped in the preparation of this manuscript on behalf of GlaxoSmithKline Biologicals.

\section{References}

1. Celentano LP, Massari M, Paramatti D, Salmaso S, Tozzi AE; EUVAC-NET Group (2005) Resurgence of pertussis in Europe. Pediatr Infect Dis J 24:761-765

2. Centers for Disease Control and Prevention (CDC) (2002) Pertussis-United States, 1997-2000. MMWR Morb Mortal Wkly Rep 51:73-76

3. Central European Vaccination Advisory Group (CEVAG) (2008) Home page at: http://www.cevag.org/index.php

4. Dilli D, Bostanci I, Dallar Y, Buzgan T, Irmak H, Torunoğlu MA (2008) Recent findings on pertussis epidemiology in Turkey. Eur J Clin Microbiol Infect Dis 27:335-341

5. Edwards KM (2005) Overview of pertussis: focus on epidemiology, sources of infection, and long term protection after infant vaccination. Pediatr Infect Dis J 24(6 Suppl):S104-S108

6. Gustafsson L, Hessel L, Storsaeter J, Olin P (2006) Long-term follow-up of Swedish children vaccinated with acellular pertussis vaccines at 3,5, and 12 months of age indicates the need for a booster dose at 5 to 7 years of age. Pediatrics 118(3):978-984

7. Gzyl A, Augustynowicz E, Rabczenko D, Gniadek G, Slusarczyk $\mathrm{J}$ (2004) Pertussis in Poland. Int J Epidemiol 33:358-365

8. Health Canada, Population and Public Health Branch. Notifiable diseases on-line. 1989-2004. Available online at: http://dsol-smed. hc-sc.gc.ca/dsol-smed/ndis/index_e.html

9. Hewlett EL, Edwards KM (2005) Clinical practice. Pertussis-not just for kids. N Engl J Med 352:1215-1222

10. Inandi T, Guraksin A, Hacialioglu N (2005) Seroprevalence of pertussis among children in Eastern Turkey. Public Health 119: $550-555$ 
11. Jogiste A, Varjas J, Jarviste A (2005) Pertussis in Estonia. Estonian Doctor 84:31-35

12. Mattoo S, Cherry JD (2005) Molecular pathogenesis, epidemiology, and clinical manifestations of respiratory infections due to Bordetella pertussis and other Bordetella subspecies. Clin Microbiol Rev 18:326-382

13. Narkeviciute I, Kavaliunaite E, Bernatoniene G, Eidukevicius R (2005) Clinical presentation of pertussis in fully immunized children in Lithuania. BMC Infect Dis 5:40

14. Tan T, Trindade E, Skowronski D (2005) Epidemiology of pertussis. Pediatr Infect Dis J 24:S10-S18

15. Torm S, Meriste S, Tamm E, Alusalu S, Järviste A, Lang K (2005) Pertussis outbreak in a basic school in Estonia: description, contributing factors and vaccine effectiveness. Scand J Infect Dis 37:664-668

16. Tozzi AE, Pandolfi E, Celentano LP, Massari M, Salmaso S, Ciofi degli Atti ML; EUVAC-NET Study Group (2007) Comparison of pertussis surveillance systems in Europe. Vaccine 25:291-297

17. Vatansever U, Cöplü N, Oner N, Sönmez C, Karasalihoglu S, Kurtoglu D, Esen B, Ekuklu G (2005) Seroprevalance of
Bordetella pertussis antibodies among healthy adolescent girls in Edirne. Swiss Med Wkly 135:531-536

18. Ward JI, Cherry JD, Chang SJ, Partridge S, Keitel W, Edwards K, Lee M, Treanor J, Greenberg DP, Barenkamp S, Bernstein DI, Edelman R; APERT Study Group (2006) Bordetella Pertussis infections in vaccinated and unvaccinated adolescents and adults, as assessed in a national prospective randomized Acellular Pertussis Vaccine Trial (APERT). Clin Infect Dis 43:151-157

19. Wirsing von König C-H, Campins-Marti M, Finn A, Guiso N, Mertsola J, Liese J (2005) Pertussis immunization in the Global Pertussis Initiative European Region: recommended strategies and implementation considerations. Pediatr Infect Dis J 24:S87-S92

20. World Health Organization (2003) WHO-recommended standards for surveillance of selected vaccine-preventable diseases. WHO/ $\mathrm{V} \& \mathrm{~B} / 03.01$. Available online at: http://www.who.int/vaccinesdocuments/DocsPDF06/843.pdf

21. World Health Organization (2008) WHO UNICEF review of national immunization coverage, 1980-2006. Available online at: http://www.who.int/immunization_monitoring/en/globalsummary/ wucoveragecountrylist.cfm\#C 